\title{
A database of natural products and chemical entities from marine habitat
}

\author{
Padavala Ajay Babu ${ }^{1 *}$, Suma Sree Puppala ${ }^{2}$, Satyavarapu Lakshmi Aswini ${ }^{2}$ Metta Ramya Vani ${ }^{2}$, \\ Chinta Narasimha Kumar ${ }^{1}$ and Tallapragada Prasanna ${ }^{1}$ \\ ${ }^{1}$ ProGene Biosciences, Institute of Bioinformatics and Research Centre, 103, Bharat Towers, Dwaraka Nagar, Visakhapatnam - 530 016, India; \\ ${ }^{2}$ Department of Biotechnology, Godavari Institute of Engineering and Technology, Rajahmundry, India; \\ Padavala Ajay Babu* - E-mail: ajay_pgb@progenebio.in; * Corresponding author \\ received September 16, 2008; accepted October 15, 2008; published November 09, 2008
}

\begin{abstract}
:
Marine compound database consists of marine natural products and chemical entities, collected from various literature sources, which are known to possess bioactivity against human diseases. The database is constructed using html code. The 12 categories of 182 compounds are provided with the source, compound name, 2-dimensional structure, bioactivity and clinical trial information. The database is freely available online and can be accessed at http://www.progenebio.in/mcdb/index.htm
\end{abstract}

Keywords: database; marine compounds; natural products

\section{Background:}

It is a known fact that water occupies at least three-quarters (3/4ths) of the earth's surface. Of this, more than $70 \%$ is the marine water. Hence, marine waters form the reservoirs for a large variety of number of organisms. Different species of organisms produce various metabolites. Several thousands of these compounds have been claimed to possess medicinal properties. Metabolites used to treat cancer or other dreadful diseases are of considerable interest for the scientific communities. The active metabolites are isolated from different marine organisms and can be used directly as drugs or as pharmaceutical agents. Several of these metabolites might offer a natural key to unlock the complications of several diseases. Because of the immense biological diversity in the sea, it has been recognized that a variety of natural products and novel chemical entities exist in oceans and that they possess biological activities with greater efficacy and specificity against human diseases [1].

Diseases like cancer, malaria, tuberculosis and several other disorders are affecting large number of people world wide. Many of the marine organism derived compounds are known to exhibit therapeutic potential and are more potent against various disorders. For example, it has been reported that cone snail venoms are highly potent and act as selective peptide antagonists or agonists of ligand or voltage-gated ion channels and G-protein-coupled receptors (GPCRs) [2]. Similarly, $\alpha$ - and $\alpha \mathrm{A}$-conotoxins, are known to competitively inhibit nicotinic acetylcholine receptors (nAChR) whereas $\psi$ conotoxins show noncompetitive inhibition of this family of receptors. Several such studies have been reported in literature [3, 4]. Information on marine compounds is of prime interest to researchers involved in drug discovery,

ISSN 0973-2063 (online) 0973-2063 (print)

Bioinformation 3(3): 142-143 (2008) natural products isolation, purification and characterization, or marine biology research. Hence, initially, we created a database of 182 natural products and chemical entities from marine habitat that contains information related to the organism, derived compound, the disease against which the compound is active and the status of clinical trials, if any.

\section{Methodology: \\ Construction of Marine Compound Database \\ The database hereafter referred as MCDB (Marine Compound Database), is constructed using html and can be accessed at http://www.progenebio.in/mcdb/index.htm. Data has been collected from various literature sources like NCBI (National Centre for Biotechnology Information) [5], Google Scholar [6], ScienceDirect [7] etc.}

\section{Features of Marine Compound Database}

MCDB contains about 182 compounds known to exhibit biological activity against human diseases are kept conveniently under 12 different categories (Table 1 in supplementary material) with number of entries under each category, given in parentheses. The additional advantage of MCDB is that the compounds from MCDB can be utilized for various scientific purposes and that these compounds, reported at a single place, could be useful in testing other diseases apart from the mentioned disease in the database, thus indicating the importance of medicinal values of the compounds. A screen-shot of the database is given in Figure1. The database architecture contains source of the compound, trade name, 2-dimensional structure, respective biological activity, clinical trial information and literature references, respectively. 


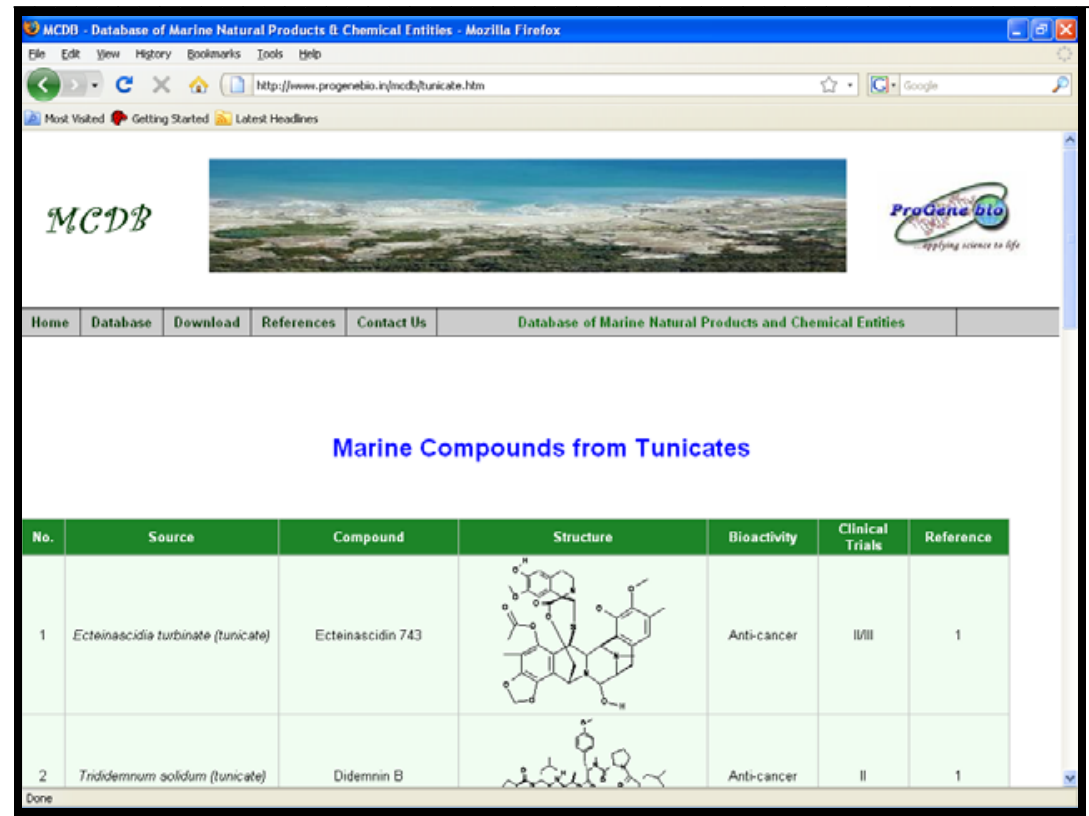

Figure 1: Screenshot image of 'Marine Compounds from Tunicates' page of MCDB.

\section{Utility:}

The database finds utility in the scientific community for a quick review of the marine organisms and their compounds for drug discovery and treatment. MCDB also contains information on clinical trials; therefore, the user shall have a quick look on the compounds that entered in any phase of clinical trials. References are provided for each compound so as to track the published data.

\section{Conclusion:}

The main objective of MCDB is to provide an easy access of marine compounds to researchers working in marine laboratories for novel drug discovery and to other users of scientific discipline. Database categorization reveals a clear mechanism of data access, and is more user-friendly. The database shall be updated periodically and released with the continuous updates of new compounds.

\section{References:}

[01] P. Proksch et al., Appl. Microbiol. Biotechnol., 59: 125 (2002) [PMID: 12111137]

[02] R. M. Jones and G. Bulaj, Curr. Pharm. Des., 6: 1249 (2000) [PMID: 10903392]

[03] B. Haefner, Drug Discov. Today, 8: 536 (2003) [PMID: 12821301]

[04] A. Kijjoa and P. Sawangwong, Mar. Drugs, 2: 73 (2004)

[05] http://www.ncbi.nlm.nih.gov

[06] www.scholar.google.com

[07] www.sciencedirect.com

Edited by P. Kangueane

Citation: Babu et al., Bioinformation 3(3): 142-143 (2008)

License statement: This is an open-access article, which permits unrestricted use, distribution, and reproduction in any medium, for non-commercial purposes, provided the original author and source are credited.

\section{Supplementary material}

Table 1: List of database categories with number of entries under each category

\begin{tabular}{llll}
\hline \multirow{2}{*}{ S. No. } & Database category (No. of entries) & S. No. & Database category (No. of entries) \\
\cline { 2 - 4 } & & & \\
\hline 1 & Sponges (101) & 7 & Bryozoans (1) \\
2 & Tunicates (13) & 8 & Worms (1) \\
3 & Coelenterates (18) & 9 & Bacteria (4) \\
4 & Echinoderms (6) & 10 & Fungi (3) \\
5 & Molluscs (16) & 11 & Ciliates (1) \\
6 & Marine Algae (15) & 12 & Fishes (3) \\
\hline
\end{tabular}

ISSN 0973-2063 (online) 0973-2063 (print)
Bioinformation, an open access forum (C) 2008 Biomedical Informatics Publishing Group 